\title{
Cardiovascular risk factors and metabolic syndrome in people with established psychotic illnesses: baseline data from the IMPaCT randomized controlled trial
}

\author{
P. Gardner-Sood ${ }^{1}$ t, J. Lally ${ }^{1,2}$ t, S. Smith ${ }^{3,4}$, Z. Atakan ${ }^{1}$, K. Ismail ${ }^{3,5}$, K. E. Greenwood ${ }^{6}$, A. Keen ${ }^{3}$, \\ C. O'Brien ${ }^{3}$, O. Onagbesan ${ }^{7}$, C. Fung ${ }^{3}$, E. Papanastasiou ${ }^{3}$, J. Eberherd ${ }^{3,8}$, A. Patel ${ }^{9}$, R. Ohlsen ${ }^{10}$, \\ D. Stahl ${ }^{11}$, A. David ${ }^{3}$, D. Hopkins ${ }^{12,13}$, R. M. Murray ${ }^{1,2}$ and F. Gaughran ${ }^{2,14 *}$ on behalf of the IMPaCT \\ team \\ ${ }^{1}$ Department of Psychosis Studies, Institute of Psychiatry, Psychology and Neuroscience (IoPPN), King's College London, London, UK \\ ${ }^{2}$ National Psychosis Service, South London and Maudsley NHS Foundation Trust, London, UK \\ ${ }^{3}$ Institute of Psychiatry, Psychology and Neuroscience (IoPPN), King's College London, London, UK \\ ${ }^{4}$ South London and Maudsley NHS Foundation Trust, London, UK \\ ${ }^{5}$ King's College Hospital NHS Foundation Trust, London, UK \\ ${ }^{6}$ School of Psychology, University of Sussex, Brighton and Early Intervention in Psychosis Service, Sussex Partnership NHS Foundation Trust, West \\ Sussex, UK \\ ${ }^{7}$ NIHR Biomedical Research Centre - BioResource for Mental Health, Social, Genetic and Development Psychiatric Centre, London, UK \\ ${ }^{8}$ Clinical Sciences, Lund University, Malmö, Sweden \\ ${ }^{9}$ Centre for the Economics of Mental and Physical Health (CEMPH), Institute of Psychiatry, Psychology and Neuroscience (IoPPN), King's College \\ London, London, UK \\ ${ }^{10}$ Florence Nightingale Faculty of Nursing and Midwifery, King's College London, London, UK \\ ${ }^{11}$ Department of Biostatistics, Institute of Psychiatry, Psychology and Neuroscience (IoPPN), King's College London, London, UK \\ ${ }^{12}$ Division of Ambulatory Care and Local Networks, King's College Hospital NHS Foundation Trust, London, UK \\ ${ }^{13}$ King's College London School of Medicine, London, UK \\ ${ }^{14}$ Institute of Psychiatry, Psychology and Neuroscience (IoPPN) and the Biomedical Research Centre, BRC Nucleus, Maudsley Hospital, South \\ London and Maudsley NHS Foundation Trust, Denmark Hill, London, UK
}

Background. The aims of the study were to determine the prevalence of cardiometabolic risk factors and establish the proportion of people with psychosis meeting criteria for the metabolic syndrome (MetS). The study also aimed to identify the key lifestyle behaviours associated with increased risk of the MetS and to investigate whether the MetS is associated with illness severity and degree of functional impairment.

Method. Baseline data were collected as part of a large randomized controlled trial (IMPaCT RCT). The study took place within community mental health teams in five Mental Health NHS Trusts in urban and rural locations across England. A total of 450 randomly selected out-patients, aged 18-65 years, with an established psychotic illness were recruited. We ascertained the prevalence rates of cardiometabolic risk factors, illness severity and functional impairment and calculated rates of the MetS, using International Diabetes Federation (IDF) and National Cholesterol Education Program Third Adult Treatment Panel criteria.

Results. High rates of cardiometabolic risk factors were found. Nearly all women and most men had waist circumference exceeding the IDF threshold for central obesity. Half the sample was obese (body mass index $\geq 30 \mathrm{~kg} / \mathrm{m}^{2}$ ) and a fifth met the criteria for type 2 diabetes mellitus. Females were more likely to be obese than males $(61 \% v .42 \%, p<0.001)$. Of the 308 patients with complete laboratory measures, $57 \%(n=175)$ met the IDF criteria for the MetS.

Conclusions. In the UK, the prevalence of cardiometabolic risk factors in individuals with psychotic illnesses is much higher than that observed in national general population studies as well as in most international studies of patients with psychosis.

Received 25 November 2014; Revised 10 March 2015; Accepted 10 March 2015; First published online 12 May 2015

Key words: Metabolic syndrome, physical health, psychotic disorder, schizophrenia, severe mental illnesses.

* Address for correspondence: F. Gaughran, PO63, Department of Psychosis Studies, Institute of Psychiatry, Psychology \& Neuroscience (IoPPN), King's College London, De Crespigny Park, London SE58AF, UK.

(Email: Fiona.1.gaughran@kcl.ac.uk)

† Joint first authors.

\section{Introduction}

The diagnosis of a psychotic illness such as schizophrenia or bipolar disorder is associated with a reduced life expectancy of 15-25 years, mostly due to increased

This is an Open Access article, distributed under the terms of the Creative Commons Attribution licence (http://creativecommons.org/ licenses/by/3.0/), which permits unrestricted re-use, distribution, and reproduction in any medium, provided the original work is properly cited. 
cardiovascular deaths (Saha et al. 2007; Kilbourne et al. 2009; Brown et al. 2010; Chang et al. 2011). A recent meta-analysis has demonstrated that the pooled relative risk of mortality among those with psychoses is 2.54 (2.35-2.75) times that of the general population, and higher rates of mortality for those with psychoses when compared with depressive and anxiety disorders (Walker et al. 2015). Cardiometabolic risk factors are common in psychosis (McEvoy et al. 2005; Leucht et al. 2007; Osborn et al. 2007; De Hert et al. 2009; Kilbourne et al. 2009). A recent meta-analysis of international studies reported prevalence rates of $44 \%$ for central obesity, $19.5 \%$ for hyperglycaemia, $10.9 \%$ for diabetes, $39 \%$ for hypertension and 39\% for dyslipidaemias, with rates increasing with age (Mitchell et al. 2013).

Clustering of cardiometabolic risk factors is termed the metabolic syndrome (MetS) (Alberti et al. 2005). Individuals with the MetS have a 3- to 6-fold increased risk of developing type 2 diabetes mellitus (Hanley et al. 2005; De Hert et al. 2011) and a 2- to 6-fold risk of mortality due to cardiovascular disease (CVD) (Hanley et al. 2005). On meta-analysis, a third of patients with schizophrenia have the MetS, with this proportion increasing with duration of illness (Mitchell et al. 2013). Slightly higher MetS rates of $34 \%$ are seen in multi-episode patients (Vancampfort et al. 2013b) while the deficit syndrome (i.e. negative symptoms of psychosis that are present as enduring traits) in schizophrenia is in itself associated with higher CVD risk (Arango et al. 2011). Similar rates of the MetS have been observed in bipolar disorder (37\%) (Vancampfort et al. 2013a) and schizo-affective disorder (27-42\%) (Basu et al. 2004; Bobes et al. 2012), although recently the Second Australian National Survey of Psychosis reported higher rates of $61 \%$ in those with psychotic disorders, suggesting an increasing prevalence with time (Morgan et al. 2014). It is possible to prevent the emergence of CVD risk in psychosis with the use of psychosocial interventions in the short term, though this is not sustained once the intervention is discontinued (Alvarez-Jimenez et al. 2010). Strategies do exist to manage established cardiometabolic risk factors (Lester et al. 2014), but despite this, a recent $\mathrm{UK}$ national audit reported screening rates for metabolic side effects of antipsychotic medication of only $11 \%$ (Barnes et al. 2007). This is further compounded by the low treatment rates of identified cardiometabolic risk factors in psychosis (Nasrallah et al. 2006). The recent Schizophrenia Commission Report (UK) highlighted the disparity of care for individuals with psychosis and emphasized the need for improved physical health intervention (Schizophrenia Commission, 2012). Encouragingly, various UK medical Royal colleges have recently agreed joint management strategies for CVD risk in psychotic illnesses (Lester et al. 2014).
In this study we aimed:

(1) To determine the prevalence of cardiometabolic risks factors and establish the proportion of people with psychosis meeting the International Diabetes Federation (IDF) criteria for the MetS.

(2) To identify the key lifestyle behaviours associated with increased risk of the MetS.

(3) To investigate whether the presence of the MetS is associated with illness severity and degree of functional impairment.

\section{Method \\ Study design}

This study used baseline data from 450 randomly selected patients with established (multi-episode) psychosis recruited as part of the National Institute for Health Research-funded study: Improving Physical Health and Reducing Substance Use in Severe Mental Illness; a randomized controlled trial (IMPaCT RCT) (Gaughran et al. 2013).

\section{Setting}

The study took place in community mental health teams (CMHTs) in five Mental Health NHS Trusts, covering urban (Lambeth, Southwark, Lewisham, Croydon, Greenwich, Bexley, Bromley) and rural (Staffordshire, Somerset and Sussex) boroughs across England (Gaughran et al. 2013).

\section{Subjects}

Potential participants were identified through their care-coordinator. All care-coordinators in participating CMHTs were approached in a random sequence and invited to participate. Patients on each participating care coordinator's caseload, meeting the inclusion criteria, were likewise approached in a random order. These patients were entered into a random numbers generator to create a randomly ordered list from which to approach potential participants. Researchers then approached these patients sequentially to participate in the RCT. In situations where a patient did not wish to take part in the study or was uncontactable, the researcher selected another patient from the list in that random order.

The inclusion criteria were as follows: aged between 18 and 65 years old; a primary diagnosis of a psychotic illness [International Classification of Diseases (ICD)-10 diagnosis: F20-29, including schizophrenia, schizo-affective disorder, bipolar affective disorder (BPAD) and delusional disorder, F31.2, F32.3 and F33.3]. The exclusion criteria included: a primary 
Table 1. Diagnostic criteria for the metabolic syndrome (International Diabetes Federation criteria) (Alberti et al. 2006)

\begin{tabular}{ll}
\hline Diagnostic criteria & \\
\hline Central obesity & \\
$\begin{array}{l}\text { Men } \\
\text { Women }\end{array}$ & Waist circumference $\geq 94 \mathrm{~cm}$ in Europid men, $\geq 90 \mathrm{~cm}$ in South Asian men \\
& women \\
Plus any two of the following: raised & $\geq 1.7 \mathrm{mmol} / 1$ or specific treatment for this lipid abnormality \\
triglycerides & \\
High-density lipoprotein-cholesterol & \\
$\begin{array}{l}\text { Men } \\
\text { Women }\end{array}$ & $<1.03 \mathrm{mmol} / 1$ \\
Raised blood pressure & $<1.29 \mathrm{mmol} / 1$ or specific treatment for this lipid abnormality \\
& Systolic blood pressure $\geq 130$ mmHg or diastolic blood pressure $\geq 85 \mathrm{mmHg}$ \\
Raised fasting plasma glucose & or treatment of previously diagnosed hypertension
\end{tabular}

diagnosis of intellectual disability (as defined by ICD-10 codes F70-F79 for intellectual disabilities); a first episode of psychosis (FEP); a primary substance misuse disorder (excluding cigarettes); a serious physical illness that could independently have an impact on metabolic measures; pregnant or up to 6 months postpartum; and receiving intensive input for a medical or terminal condition. Of patients screened, 39\% (1043/ 2663) met the inclusion criteria, with the majority excluded for not meeting the diagnostic inclusion criterion (though the exact percentage excluded by each exclusion criterion is not known).

\section{Clinical and sociodemographic variables}

Sociodemographic and clinical data including gender, age, ethnicity, diagnosis and self-reported duration of illness were collected. Diagnoses were based on ICD-10 diagnostic criteria and were extracted from the documented diagnosis in the clinical notes at the time of recruitment. Participants' mental health status was measured using the Positive And Negative Syndrome Scale (PANSS; Kay et al. 1989), the Global Assessment of Functioning (GAF; American Psychiatric Association, 2002) and the Montgomery-Åsberg Depression Rating Scale (MADRS; Montgomery \& Åsberg, 1979). Current alcohol, smoking and cannabis use was recorded using the following measures: the Alcohol Use Disorders Identification Test (AUDIT; Saunders et al. 1993); the Nicotine Dependence Questionnaire (Fagerstrom, 1978; Fagerstrom \& Schneider, 1989); Timeline Follow-Back (Sobell \& Sobell, 1992); and a urine drug screen. Total scores were derived from individual scale items. If any individual items were missing, then the total score was treated as missing. Data for all measures were collected through face-to-face interviews conducted by trained research assistants.

\section{Cardiometabolic risk factors}

Height, weight and blood pressure (BP) were measured using standardized techniques. The BP values presented are the second BP measurements, taken after an interval of $5 \mathrm{~min}$. Waist circumference was measured at the umbilicus with the patient standing. As both serum glucose and triglycerides are affected by recent food ingestion, we took fasting blood samples.

Obesity was defined using the World Health Organization reference standard [body mass index $(\mathrm{BMI}) \geq 30 \mathrm{~kg} / \mathrm{m}^{2}$ ]. Diabetes was diagnosed based on measured glycated haemoglobin (HbA1c) of $\geq 48$ $\mathrm{mmol} / \mathrm{mol}(\geq 6.5 \%)$ or fasting glucose $\geq 7.0 \mathrm{mmol} / \mathrm{l}$ or a prior diagnosis of diabetes. In addition, in keeping with recent guidance, an $\mathrm{HbA} 1 \mathrm{c}$ of $42-46 \mathrm{mmol} / \mathrm{mol}$ (6-6.4\%) was taken to indicate glucose dysregulation or a high risk of diabetes.

The IDF definitions of other cardiometabolic risk factors and of the MetS were used (see Table 1) (Alberti et al. 2005, 2006). However, we extended the definition of the MetS to include an $\mathrm{HbA} 1 \mathrm{c}$ value $\geq 42 \mathrm{mmol} / \mathrm{mol}$ $(\geq 6 \%)$, as this is consistent with a raised fasting plasma glucose $\geq 5.6 \mathrm{mmol} / 1$ (The International Expert Committee, 2009; Lester et al. 2014).

Additionally, we also determined if each patient met the adapted National Cholesterol Education Program (NCEP) Third Adult Treatment Panel (ATP-III) criteria (NCEP ATP-III criteria) (Grundy et al. 2005), to allow for comparison with selected studies. The ATP-III classification requires three of five criteria to be met for a diagnosis of the MetS and an abnormal waist circumference is not obligatory. In comparison with the IDF MetS criteria, the ATP-III criteria have a higher threshold for abnormal waist circumference, with $102 \mathrm{~cm}$ for males and $88 \mathrm{~cm}$ for females being the cut-offs. The other four criteria are comparable with the IDF criteria. 
Table 2. Clinical characteristics of the study population $(n=450)$

\begin{tabular}{lccc}
\hline & Total & Males $(n=257)$ & Females $(n=193)$ \\
\hline Mean age, years (S.D.) $(n=449)$ & $43.6(10.1)$ & $42.7(9.9)$ & $44.8(10.2)$ \\
Mean duration of illness, years (S.D.) $(n=358)$ & $15.7(10.3)$ & $16.5(10.3)$ & $14.7(10.3)$ \\
Mean PANSS total score (S.D.) $(n=417)$ & $51.2(14.1)$ & $51.5(14.3)$ & $50.9(13.9)$ \\
Mean PANSS positive symptom scale score (S.D.) $(n=421)$ & $11.8(5.0)$ & $12.0(5.1)$ & $11.5(4.8)$ \\
Mean PANSS negative symptom scale score (S.D.) $(n=418)$ & $12.9(4.9)$ & $13.1(4.9)$ & $12.6(5.0)$ \\
Mean MADRS score (s.D.) $(n=427)$ & $11.0(9.5)$ & $10.6(9.6)$ & $11.6(9.2)$ \\
Mean GAF score (s.D.) $(n=425)$ & $59.2(13.1)$ & $59.0(13.6)$ & $59.9(12.5)$ \\
Diagnosis, $n$ (\%) $(n=423 ; 245$ males, 178 females) & & & 108 \\
Schizophrenia & $299(71)$ & 191 & 37 \\
Schizo-affective disorder & $56(13)$ & 19 & 24 \\
Bipolar affective disorder & $52(12)$ & 28 & 6 \\
Depression & $11(3)$ & 5 & 3 \\
Delusional disorder & $5(1)$ & 2 & \\
\hline
\end{tabular}

S.D., Standard deviation; PANSS, Positive And Negative Syndrome Scale; MADRS, Montgomery-Åsberg Depression Rating Scale; GAF, Global Assessment of Functioning.

Hypertension was defined on the basis of a measured BP of either a systolic measure of $130 \mathrm{mmHg}$ and/or a diastolic measure of $85 \mathrm{mmHg}$ or existing treatment with antihypertensive medication.

The Dietary Instrument for Nutrition Education (DINE; Roe et al. 1994) was used to assess the dietary patterns of participants over the past week. Respondents were classified according to their saturated fat, unsaturated fat and fibre intake, based on self-reported consumption of various foods. The International Physical Activity Questionnaire was used to measure the intensity of physical activity (high, moderate, low) and the duration of physical activities over the previous week (Craig et al. 2003).

\section{Statistical analysis}

Statistical analyses were performed using the IBM Statistical Package for Social Sciences Statistics for Windows, version 20.0 (USA). Descriptive measures were used for the basic demographic and clinical variables as well as for variables relating to the evaluation of metabolic dysregulation. Student's $t$ test for parametric data and the $\chi^{2}$ test for categorical variables were employed. All statistical tests were two-sided and a $p$ value $\leq 0.05$ was considered statistically significant.

\section{Ethical Standards}

Ethical approval for this study was obtained from The Joint South London and Maudsley and The Institute of Psychiatry NHS Research Ethics Committee. Ethical approval was granted on 17 July 2009 (REC ref. no. 09/H080/41).

\section{Results}

The clinical characteristics of the study population are shown in Table 2. Mean age was 43.6 years (s.D. $=10.1$ ) and mean duration of illness was 15.7 years (s.D. = $10.3) ; 57 \%(n=257)$ were male. In respect of ethnicity, $55 \%(n=239)$ were of Caucasian ethnicity, while 33\% $(n=143)$ were of black African or black Caribbean ethnicity, with $7 \%(n=27)$ of Asian ethnicity and 5\% $(n=$ 22) of mixed ethnicity.

Only $6 \%(n=25)$ of the study population were not treated with antipsychotic medication. Of the participants, $30 \%(n=127)$ were prescribed clozapine and $16 \%(n=70)$ olanzapine. Of the participants, $75 \%$ $(n=321)$ were treated with second-generation (atypical) antipsychotics (including clozapine and olanzapine), $16 \%(n=71)$ were treated with first-generation antipsychotics and $8 \%(n=36)$ were treated with a combination of antipsychotics.

\section{Cardiovascular risk factors (see Table 3)}

Of the subjects, 48\% (192/398) were obese (BMI > $30 \mathrm{~kg} /$ $\mathrm{m}^{2}$ ). Female patients had significantly higher BMIs than males $[t=2.329$, degrees of freedom $(\mathrm{df})=426$, $p<0.020]$.

In all, 83\% had abdominal obesity, with 95\% (160/ 169) of females meeting the IDF criterion for central obesity, significantly higher than males $(74 \%$, $n=167 / 227)\left(\chi^{2}=29.994, p<0.0001\right)$. Of the participants, $89 \%$ (305/342) had evidence of dyslipidaemia, defined as the presence of at least one abnormal lipid parameter (high total cholesterol, low high-density lipoprotein-cholesterol, high triglyceride) $(n=233)$ or treatment with lipid-lowering drugs $(n=72)$. Sixty-six 


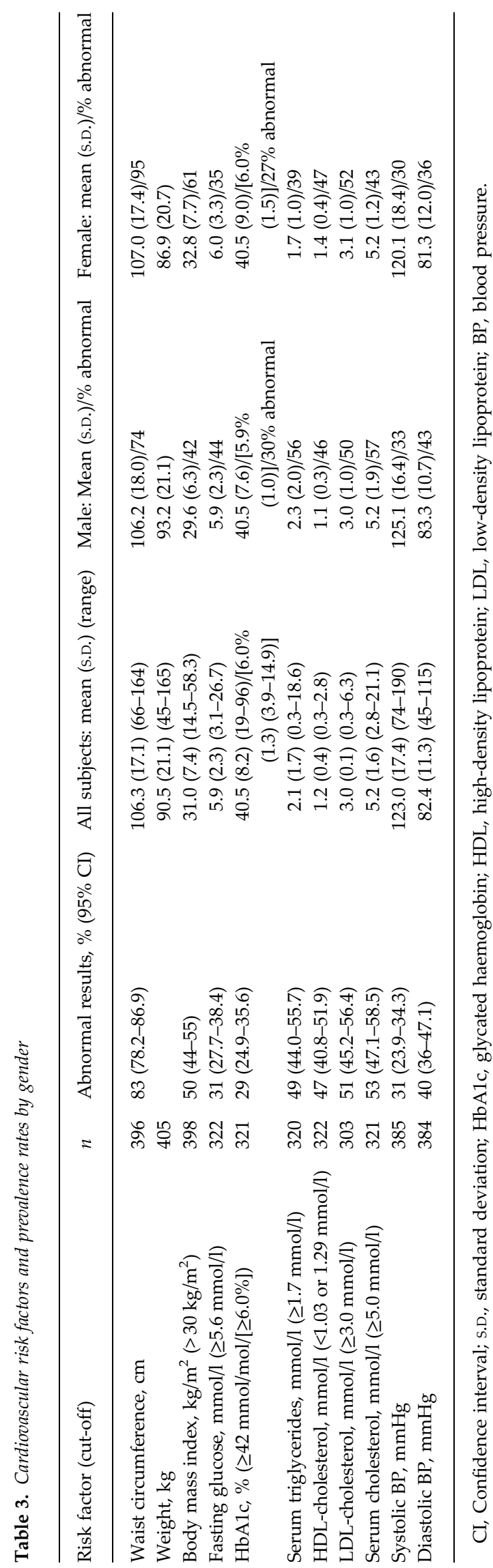

people out of the $330(20 \%)$ for whom we had data regarding either previous diagnosis of diabetes mellitus $(n=38)$ and/or had relevant blood results $(n=28)$ met the criteria for type 2 diabetes mellitus. An additional $85 / 286(30 \%)$ had evidence of glucose dysregulation on their blood tests. Hypertension was present in 214/398 (54\%), of whom 62 were on anti-hypertensive therapy. Nearly half $(49 \%, n=195 / 398)$ were hypertensive on measurement. Of the subjects, $62 \%$ (268/432) smoked tobacco, smoking an average of 18.2 (S.D. = 11.6) cigarettes per day.

There was no significant difference between mean BMI $(p=0.241)$ and waist circumference $(p=0.437)$ when stratified by geographical location (by borough).

The prescription of either olanzapine or clozapine $(n=191)$ (which are the antipsychotic medications traditionally associated with the highest risk of metabolic dysfunction) was not associated with an increased rate of obesity $(n=80,42 \%)$ compared with those not prescribed either dibenzodiazepine $(n=113,54 \%)$ $\left(\chi^{2}=1.270, \mathrm{df}=1, p=0.153\right)$. When clozapine alone $(n=117)$ was compared with other antipsychotics (excluding olanzapine), no excess in obesity was found on clozapine $(47 \%, n=55)$ versus other antipsychotics $(52 \%, n=99)\left(\chi^{2}=0.675, p=0.241\right)$. Also, there was no excess of other cardiometabolic risk factors [dyslipidaemia $(p=0.418)$, hypertension $(p=0.546)$ and diabetes $(p=0.463)]$.

\section{Ethnicity}

Those of white ethnicity had larger mean waist circumferences $($ mean $=108.5 \mathrm{~cm}$, S.D. $=17.0$ ) than those of black African or Caribbean ethnicity (mean $=104.4$ $\mathrm{cm}, \quad$ S.D. $=16.9)(t=2.225, \mathrm{df}=345, p=0.027)$. White patients had higher serum triglycerides than black African or Caribbean patients (mean difference $=0.89$, $p<0.003$ ). However, there were no significant differences in overall rates of dyslipidaemia $(p=0.545)$, hypertension $(p=0.101)$, diabetes $(p=0.298)$ and obesity $\left(\mathrm{BMI}>30 \mathrm{~kg} / \mathrm{m}^{2}\right) \quad(p=0.140)$. By gender, females of black African or Caribbean ethnicity had significantly higher rates of obesity $(n=28 / 37)$ as measured by BMI than females of white ethnicity $(n=35 / 63)\left(\chi^{2}=\right.$ 4.048, $p=0.035$ ).

\section{MetS}

Of the 450 participants, there were 308 patients with complete laboratory and clinical measures to allow MetS status to be confirmed. In this group, the prevalence of IDF MetS was $56.8 \%(n=175 / 308)$. The prevalence of the MetS as defined by the ATP-III criteria was $56.2 \%(n=173 / 308)$. Those with IDF MetS were older (mean age $=44.68$ years, S.D. $=9.8)$ than those without IDF MetS (42.12 years, S.D. $=10.4)(t=-2.28, p=0.028)$. 
There was no difference in duration of illness ( $p=0.358)$, or the use of clozapine $(p=0.282)$ or olanzapine $(p=0.303)$ in those with IDF MetS compared with those without. White patients had higher rates of IDF MetS $(n=114 / 171)$ than those of black African or Caribbean ethnicity $(n=47 / 101)\left(\chi^{2}=10.654, p=0.001\right)$, with this most pronounced in white males who had significantly higher rates of IDF MetS $(n=66 / 96)$ than males of black African or Caribbean heritage $(n=29 / 63)\left(\chi^{2}=8.143, p=0.004\right)$.

Of the 13 individuals who were not prescribed antipsychotics and who had complete measures to allow for MetS status to be confirmed, eight met the criteria for IDF MetS.

\section{Psychopathology and cardiometabolic risk factors (see Table 4)}

A consistent relationship was found between cardiometabolic risk factors and functional impairment as measured by GAF scores. Degree of psychosis as measured by the PANSS and subscale scores was not significantly associated with any individual cardiometabolic risk factor. The only individual cardiometabolic risk factor associated with depressive symptomatology (MADRS) was obesity $(p=0.032)$

There was no relationship between duration of psychotic illness and any of obesity (BMI $\left.>30 \mathrm{~kg} / \mathrm{m}^{2}\right)$ $(t=0.410, \mathrm{df}=329, p=0.682)$, hypertension $(t=-1.346$, $p=0.179)$, dyslipidaemia $(t=-0.144, p=0.886)$ or presence of diabetes mellitus $(t=-0.992, \mathrm{df}=251, p=0.322)$.

\section{Drug and alcohol use}

Of the respondents, $28 \%(n=68 / 291)$ had hazardous/ harmful alcohol use (AUDIT score $\geq 8$ ), while the alcohol intake of a further $4 \%(n=12 / 291)$ indicated the need for diagnostic evaluation for alcohol dependence syndrome (AUDIT score $\geq 20$ ).

Of the respondents, $18 \%(n=76 / 422)$ were current cannabis users, with 52 people identified through the Timeline Follow-Back procedure and a further 24 identified through a cannabinoid-positive urinary drug screen.

\section{Other substance use}

Of the participants, $3 \%(n=13 / 439)$ were cocaine or amphetamine users, $1.4 \% \quad(n=6)$ reported ongoing crack cocaine use and less than $1 \%(n=3)$ reported using ecstasy.

\section{Lifestyle measures}

Of the participants, 23\% (102/435) had a high saturated fat intake score on the DINE, 29.7\% (129/435) had a medium fat intake, while $47 \%(204 / 435)$ had a low

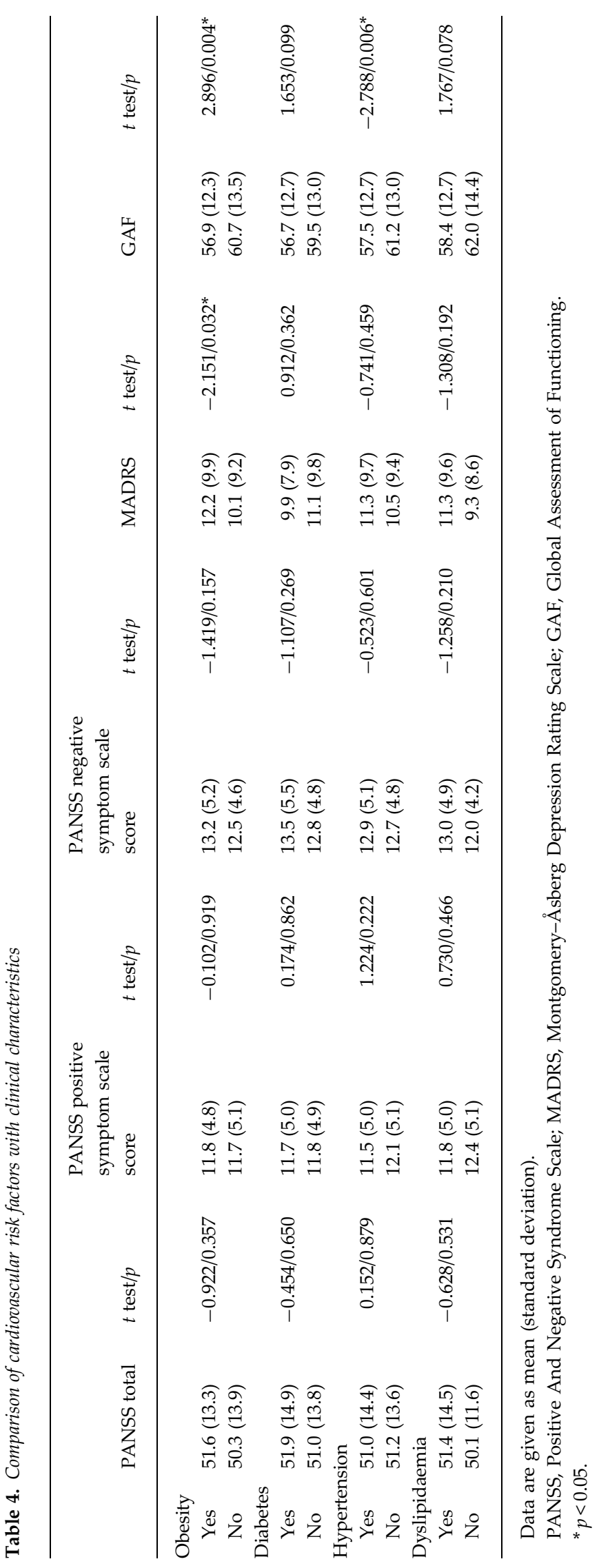


saturated fat intake; $51 \%$ of the participants $(n=210)$ had a low fibre intake, with only $25 \%(n=102)$ reporting a high fibre intake.

Of the participants, $44 \% \quad(n=196)$ were engaging in low-intensity physical activity, $44 \%(n=200)$ engaged in moderate-intensity physical activity, while $12 \%$ $(n=54)$ participated in high-intensity physical activity. Engaging in high levels of physical activity was associated with lower rates of obesity $\left(\chi^{2}=20.944\right.$, $p<0.0001)$ and dyslipidaemia $\left(\chi^{2}=17.537, p=0.001\right)$ compared with low-intensity physical activity. Further, lower rates of the MetS were found in those with high-intensity physical activity $(n=15 / 41)$, compared with those engaging in low-intensity physical activity $(n=160 / 267)\left(\chi^{2}=7.891, p=0.004\right)$.

\section{Discussion}

This cohort of individuals with established psychosis had a high prevalence of both individual cardiometabolic risk factors and of the MetS. Rates of central obesity were alarmingly high at $83 \%$, with almost all women $(95 \%)$ exceeding the IDF threshold. Half the sample were obese, half had hypertriglyceridaemia and $54 \%$ were hypertensive. Of the study population, $50 \%$ had evidence of glucose dysregulation, including $20 \%$ of the sample with type 2 diabetes, which is much higher than the rate in the general UK population (International Diabetes Federation, 2013). This excess is amplified when an aged-matched comparison is used; the expected prevalence of diabetes in UK residents with an age range from 35 to 54 years would be $9.4 \%$ for men and $6.6 \%$ for women (Craig \& Mindell, 2011).

Of our participants, $56.8 \%$ met criteria for the MetS, greatly exceeding the international figure of $34 \%$ described in a recent meta-analysis of the MetS in schizophrenia (Mitchell et al. 2013). This meta-analysis found a lack of homogeneity in the distribution of MetS prevalence across individual studies. Similar rates of cardiometabolic risk to what we found have been identified in a large Australian prevalence survey in psychosis, where rates of abdominal obesity greater than $80 \%$ were found, as well as comparable rates of the MetS, dyslipidaemia and hypertension (Galletly et al. 2012). Our prevalence rates thus place this UK population at the higher end globally for cardiometabolic risk factors in psychosis. Given that UK general population studies have identified rates of the MetS of 25$34 \%$, the almost doubled rate in psychosis is striking (Khunti et al. 2010; Langan et al. 2012) and behoves us to investigate the antecedents and remedies. Consistent with general population studies, but in contrast to some cross-sectional studies in psychosis, we found no gender difference in the rates of the MetS (Ford, 2005), although we did find higher rates of obesity (as defined by BMI) in females (61\% v. 42\%) (McEvoy et al. 2005; Bobes et al. 2007). There were ethnic differences in rates of the MetS, with those of white ethnicity showing higher rates compared with those of black African or Caribbean ethnicity, in keeping with previous work (McEvoy et al. 2005; Keenan et al. 2013), results which have not been consistently demonstrated in the general population (Keenan et al. 2013).

Lifestyle choices amplify the potential consequences of abnormal metabolic parameters in this population: $62 \%$ were smokers, hugely in excess of the current smoking rates in the general UK population of $20 \%$ (Health \& Social Care Information Centre, 2013). A recent 11-year follow-up of 517 people with schizophrenia showed an overall standardized mortality ratio of 2.80 , smoking being a very strong predictor of death (relative risk of 4.66) (Dickerson et al. 2014). Smoking not only increases the risk of early death, but also complicates treatment, in that the hydrocarbons in cigarette smoke accelerate the metabolism of dibenzodiazepines, clozapine and olanzapine (Rostami-Hodjegan et al. 2004). Until recently, clinicians have shown little interest in supporting smoking cessation in psychosis patients. However, findings from a systematic review have demonstrated that people with schizophrenia want to stop smoking and that it is possible to help them to do so (Banham \& Gilbody, 2010) and it is logical that this should now be a priority public health measure.

Lack of exercise was common among the study population, with only $12 \%$ of individuals participating in high-intensity physical activity, consistent with previous reports (Faulkner et al. 2006), with high-intensity physical activity associated with lower rates of obesity and dyslipidaemia. As exercise is linked to greater longevity in the general population (Paffenbarger et al. 1993; Manson et al. 2002), it is likely that greater emphasis on engaging in physical activity among those with severe mental illnesses (SMI) would help reduce mortality risk (Vancampfort et al. 2010). This will require addressing the individual barriers to regular physical activity and promoting it within the structure of community treatment programmes (Vancampfort et al. 2012) but will also entail addressing wider organizational barriers to regular physical activity and other health promotion programmes (O’Brien et al. 2014).

We did not identify any associations between individuals' psychopathology scores on PANSS and their cardiometabolic risk. However, we did find associations between increased functional impairment and obesity, diabetes and hypertension and between higher depression scores and obesity. Of note, in the US Clinical Antipsychotic Trials of Intervention Effectiveness (CATIE) study, the PANSS mean total score of 75.9 was higher than in IMPaCT (51.2) - with lower NCEP ATP-III MetS rates of $36 \%$ in CATIE compared with 
the ATP-III MetS rate of $56 \%$ in IMPaCT, meaning that our higher rates of cardiometabolic risk are not a result of illness severity (Meyer et al. 2005).

The rate of the MetS in the BPAD subgroup was $66 \%$, which is higher than previously reported rates of 22-30\% (Garcia-Portilla et al. 2008; van Winkel et al. 2008). This may relate more to service bias than diagnostic factors. Patients with bipolar disorder requiring ongoing secondary care management in the UK may have a greater clinical need than those managed in primary care and as we have demonstrated an effect of global function on cardiometabolic risk, this is one potential explanation, although the numbers were too small to test this hypothesis.

Despite the mean duration of illness being 15.7 years, we found no effect of duration of illness on the prevalence of the MetS, in contrast to the meta-analysis conducted by Mitchell et al. (2013). Even though our study excluded, for pragmatic reasons, those people registered with early intervention services, which manage younger people for up to 3 years from first presentation, we would have expected to find an effect.

A high proportion of this sample was prescribed dibenzodiazepines, which are associated with early emergence of cardiometabolic risk factors (Howes et al. 2004; Nielsen et al. 2010). However, we found no differences in cardiac risk factors between those on clozapine or olanzapine and those not. One potential explanation is that the cardiometabolic effect plateaus and becomes more evenly distributed over a more prolonged course of illness. However, as we do not have access to treatment histories, it may also be that this homogeneity across medications reflects the cumulative effects of medication changes over the years. This lack of difference is especially interesting in light of the evidence that patients with schizophrenia treated with clozapine have the least reduction in life expectancy of all patients with schizophrenia (Tiihonen et al. 2009; Hayes et al. 2014).

Strengths of this study include a sample of randomly selected individuals with psychosis from diverse ethnic backgrounds and the mix of urban and rural locations adding to the generalizability of the study findings. Limitations of the study include the crosssectional design, and the lack of a control group. We have not adjusted for the effect of socio-economic deprivation. The sample was recruited from across England, but predominantly from Greater London, including some boroughs with high levels of deprivation, though we identified no difference in metabolic variables by borough. A recent Scottish paper noted an excess of mortality in SMI linked to deprivation, although this effect was not evident for deaths due to cerebrovascular disease or CVD (Langan Martin et al. 2014). The missing data from metabolic measures and clinical assessment scales may have introduced a selection bias into the study, but it is not obvious what effect such a bias would result in. Measures such as duration of illness were selfreported and thus are non-validated. We have no data on duration of antipsychotic treatment and previous antipsychotic treatments used over the longitudinal course of an individual's illness, both of which are confounding factors that could potentially make an impact on an individual's cardiometabolic risk factors. The exclusion of FEP patients from this study recognizes the different CVD profiles in the early stages of psychosis. Data on the evolution of CVD risk factors from first presentation are being gathered as part of a different project within IMPaCT. To have included FEP data within this study would have underestimated the extent of the problem which patients with established psychosis, their carers and their clinicians face.

Because of the design of the study, individuals with established psychosis cared for by their general practitioners were not included in the study population. This group is estimated to make up $30 \%$ of all patients with SMI in the UK (Reilly et al. 2012). Our study population, compared with this primary care-only population, may be functionally or symptomatically more impaired; and antipsychotic prescribing patterns may be different - for instance, clozapine is rarely prescribed in primary care. Thus the study's findings may not necessarily generalize to those patients living in the community who share these same diagnoses but who are not managed in secondary care.

\section{Conclusion}

The results from this study are alarming and draw attention to the huge rates of modifiable cardiometabolic risk factors in people with established psychosis in the UK. The prevalence of the MetS in people with established psychosis in this UK sample is $56.8 \%$. The main defining measure of the MetS, waist circumference, exceeded the IDF diagnostic cut-off in $83 \%$ of our patients, and in $95 \%$ of female patients. Although central obesity is known to be the best predictor of morbidity and mortality (Zimmet et al. 2001), waist circumference has not to date been routinely measured in people with psychosis, although it has now been included in the latest National Institute of Health and Clinical Excellence (2014) guidelines for schizophrenia. Practice in in-patient psychiatric services has improved with the advent of incentivization and of shared guidelines for CVD risk management in psychosis such as the Lester Cardiometabolic Health Resource (Lester et al. 2014). However, serious shortcomings remain in the management of physical health care in community-based patients, as evidenced by the National Audit of Schizophrenia findings (NAS, 2012; Crawford 
et al. 2014). Nevertheless, more tailored work is needed if we wish to improve life expectancy in psychosis, with these findings reinforcing the need for routine monitoring of waist circumference in psychosis and assertive management of cardiovascular risk in people with psychosis.

\section{Acknowledgements}

The National Institute for Health Research funds the $\mathrm{IMPaCT}$ programme at King's College London and the South London and Maudsley NHS Foundation Trust (ref. RP-PG-0606-1049). The funding source(s) had no role in the design of this study and did not have any role during its execution, analyses, interpretation of the data, or decision to submit results. We affirm that the paper is an honest, accurate and transparent account of the study being reported; that no important aspects of the study have been omitted; and that any discrepancies from the study as planned (and, if relevant, registered) have been explained.

The study is registered with ISRCTN (International Standard Randomised Controlled Trial Number) as ISRCTN58667926.

\section{Declaration of Interest}

R.M.M. has received payment for lectures including service on speakers' bureaus for BMS, Janssen and AZ. J.E. is an international medical manager at and employee of H. Lundbeck. F.G. has received honoraria for advisory work and lectures from Roche, BMS, Lundbeck and Sunovion, and has a family member with professional links to Lilly and GSK. The other authors have no financial relationships with any organizations that might have an interest in the study in the previous 3 years. There are no other relationships or activities that could appear to have influenced the study.

\section{References}

Alberti KG, Zimmet P, Shaw J (2005). The metabolic syndrome - a new worldwide definition. Lancet 366, 1059-1062.

Alberti KG, Zimmet P, Shaw J (2006). Metabolic syndrome a new world-wide definition. A Consensus Statement from the International Diabetes Federation. Diabetic Medicine 23, 469-480.

Alvarez-Jimenez M, Martinez-Garcia O, Perez-Iglesias R, Ramirez ML, Vazquez-Barquero JL, Crespo-Facorro B (2010). Prevention of antipsychotic-induced weight gain with early behavioural intervention in first-episode psychosis: 2-year results of a randomized controlled trial. Schizophrenia Research 116, 16-19.

American Psychiatric Association (2002). Multiaxial assessment. In Diagnostic and Statistical Manual of Mental
Disorders, 4th edn, text revised (DSM-IV-TR). APA: Arlington, VA.

Arango C, Bobes J, Kirkpatrick B, Garcia-Garcia M, Rejas J (2011). Psychopathology, coronary heart disease and metabolic syndrome in schizophrenia spectrum patients with deficit versus non-deficit schizophrenia: findings from the CLAMORS study. European Neuropsychopharmacology 21, 867-875.

Banham L, Gilbody S (2010). Smoking cessation in severe mental illness: what works? Addiction 105, 1176-1189.

Barnes TR, Paton C, Cavanagh MR, Hancock E, Taylor DM (2007). A UK audit of screening for the metabolic side effects of antipsychotics in community patients. Schizophrenia Bulletin 33, 1397-1403.

Basu R, Brar JS, Chengappa KN, John V, Parepally H, Gershon S, Schlicht P, Kupfer DJ (2004). The prevalence of the metabolic syndrome in patients with schizoaffective disorder-bipolar subtype. Bipolar Disorders 6, 314-318.

Bobes J, Arango C, Aranda P, Carmena R, Garcia-Garcia M, Rejas J (2007). Cardiovascular and metabolic risk in outpatients with schizophrenia treated with antipsychotics: results of the CLAMORS Study. Schizophrenia Research 90, 162-173.

Bobes J, Arango C, Aranda P, Carmena R, Garcia-Garcia M, Rejas J (2012). Cardiovascular and metabolic risk in outpatients with schizoaffective disorder treated with antipsychotics: results from the CLAMORS study. European Psychiatry 27, 267-274.

Brown S, Kim M, Mitchell C, Inskip H (2010). Twenty-five year mortality of a community cohort with schizophrenia. British Journal of Psychiatry 196, 116-121.

Chang C-K, Hayes RD, Perera G, Broadbent MTM, Fernandes AC, Lee WE, Hotopf M, Stewart R (2011). Life expectancy at birth for people with serious mental illness and other major disorders from a secondary mental health care case register in London. PLoS ONE 6, e19590.

Craig CL, Marshall AL, Sjostrom M, Bauman AE, Booth ML, Ainsworth BE, Pratt M, Ekelund U, Yngve A, Sallis JF, Oja P (2003). International Physical Activity Questionnaire: 12-country reliability and validity. Medicine and Science in Sports and Exercise 35, 1381-1395.

Craig R, Mindell J (2011). Health Survey for England 2010. The Information Centre: London.

Crawford MJ, Jayakumar S, Lemmey SJ, Zalewska K, Patel MX, Cooper SJ, Shiers D (2014). Assessment and treatment of physical health problems among people with schizophrenia: national cross-sectional study. British Journal of Psychiatry 205, 473-477.

De Hert M, Correll CU, Bobes J, Cetkovich-Bakmas M, Cohen D, Asai I, Detraux J, Gautam S, Moller HJ, Ndetei DM, Newcomer JW, Uwakwe R, Leucht S (2011). Physical illness in patients with severe mental disorders. I. Prevalence, impact of medications and disparities in health care. World Psychiatry 10, 52-77.

De Hert M, Dekker JM, Wood D, Kahl KG, Holt RI, Moller HJ (2009). Cardiovascular disease and diabetes in people with severe mental illness position statement from the European Psychiatric Association (EPA), supported by the European Association for the Study of Diabetes (EASD) and the European Society of Cardiology (ESC). European Psychiatry 24, 412-424. 
Dickerson F, Stallings C, Origoni A, Schroeder J, Khushalani S, Yolken R (2014). Mortality in schizophrenia: clinical and serological predictors. Schizophrenia Bulletin 40, 796-803.

Fagerstrom KO (1978). Measuring degree of physical dependence to tobacco smoking with reference to individualization of treatment. Addictive Behaviors 3, 235-241.

Fagerstrom KO, Schneider NG (1989). Measuring nicotine dependence: a review of the Fagerstrom Tolerance Questionnaire. Journal of Behavioral Medicine 12, 159-182.

Faulkner G, Cohn T, Remington G (2006). Validation of a physical activity assessment tool for individuals with schizophrenia. Schizophrenia Research 82, 225-231.

Ford ES (2005). Prevalence of the metabolic syndrome defined by the International Diabetes Federation among adults in the U.S. Diabetes Care 28, 2745-2749.

Galletly CA, Foley DL, Waterreus A, Watts GF, Castle DJ, McGrath JJ, Mackinnon A, Morgan VA (2012).

Cardiometabolic risk factors in people with psychotic disorders: the Second Australian National Survey of Psychosis. Australian and New Zealand Journal of Psychiatry 46, 753-761.

Garcia-Portilla MP, Saiz PA, Benabarre A, Sierra P, Perez J, Rodriguez A, Livianos L, Torres P, Bobes J (2008). The prevalence of metabolic syndrome in patients with bipolar disorder. Journal of Affective Disorders 106, 197-201.

Gaughran F, Stahl D, Ismail K, Atakan Z, Lally J, Gardner-Sood P, Patel A, David A, Hopkins D, Harries B, Lowe P, Orr D, Arbuthnot M, Murray R, Greenwood K, Smith S (2013). Improving Physical Health and Reducing Substance Use in Psychosis - randomised control trial (IMPaCT RCT): study protocol for a cluster randomised controlled trial. BMC Psychiatry 13, 263.

Grundy SM, Cleeman JI, Daniels SR, Donato KA, Eckel RH, Franklin BA, Gordon DJ, Krauss RM, Savage PJ, Smith SC Jr, Spertus JA, Costa F (2005). Diagnosis and management of the metabolic syndrome: an American Heart Association/National Heart, Lung, and Blood Institute Scientific Statement. Circulation 112, 2735-2752.

Hanley AJG, Karter AJ, Williams K, Festa A, D'Agostino RB, Wagenknecht LE, Haffner SM (2005). Prediction of type 2 diabetes mellitus with alternative definitions of the metabolic syndrome: The Insulin Resistance Atherosclerosis Study. Circulation 112, 3713-3721.

Hayes RD, Downs J, Chang C-K, Jackson RG, Shetty H, Broadbent M, Hotopf M, Stewart R (2014). The effect of clozapine on premature mortality: an assessment of clinical monitoring and other potential confounders. Schizophrenia Bulletin. Published online 25 August 2014. doi:10.1093/ schbul/sbu120.

Health \& Social Care Information Centre (2013). Statistics on Smoking: England, 2013. Health \& Social Care Information Centre: Cardiff.

Howes OD, Gaughran FP, Amiel SA, Murray RM, Pilowsky LS (2004). The effect of clozapine on factors controlling glucose homeostasis. Journal of Clinical Psychiatry 65, 1352-1355.

International Diabetes Federation (2013). IDF Diabetes Atlas. International Diabetes Federation: Brussels, Belgium.
Kay SR, Opler LA, Lindenmayer JP (1989). The Positive and Negative Syndrome Scale (PANSS): rationale and standardisation. British Journal of Psychiatry Supplement 7, 59-67.

Keenan TE, Yu A, Cooper LA, Appel LJ, Guallar E, Gennusa JV III, Dickerson FB, Crum RM, Anderson CA, Campbell LM, Young DR, Daumit GL (2013). Racial patterns of cardiovascular disease risk factors in serious mental illness and the overall U.S. population. Schizophrenia Research 150, 211-216.

Khunti K, Taub N, Tringham J, Jarvis J, Farooqi A, Skinner TC, Davies MJ (2010). Screening for the metabolic syndrome using simple anthropometric measurements in south Asian and white Europeans: a population-based screening study. The Leicester Ethnic Atherosclerosis and Diabetes Risk (LEADER) Study. Primary Care Diabetes 4, 25-32.

Kilbourne AM, Morden NE, Austin K, Ilgen M, McCarthy JF, Dalack G, Blow FC (2009). Excess heart-disease-related mortality in a national study of patients with mental disorders: identifying modifiable risk factors. General Hospital Psychiatry 31, 555-563.

Langan SM, Seminara NM, Shin DB, Troxel AB, Kimmel SE, Mehta NN, Margolis DJ, Gelfand JM (2012). Prevalence of metabolic syndrome in patients with psoriasis: a population-based study in the United Kingdom. Journal of Investigative Dermatology 132, 556-562.

Langan Martin J, McLean G, Park J, Martin DJ, Connolly M, Mercer SW, Smith DJ (2014). Impact of socioeconomic deprivation on rate and cause of death in severe mental illness. BMC Psychiatry 14, 261.

Lester H, Shiers D, Rafi I, Cooper S, Holt R (2014). Positive Cardiometabolic Health Resource: an Intervention Framework for Patients with Psychosis on Antipsychotic Medication. Royal College of Psychiatrists: London.

Leucht S, Burkard T, Henderson J, Maj M, Sartorius N (2007). Physical illness and schizophrenia: a review of the literature. Acta Psychiatrica Scandinavica 116, 317-333.

Manson JE, Greenland P, LaCroix AZ, Stefanick ML, Mouton CP, Oberman A, Perri MG, Sheps DS, Pettinger MB, Siscovick DS (2002). Walking compared with vigorous exercise for the prevention of cardiovascular events in women. New England Journal of Medicine 347, 716-725.

McEvoy JP, Meyer JM, Goff DC, Nasrallah HA, Davis SM, Sullivan L, Meltzer HY, Hsiao J, Scott Stroup T, Lieberman JA (2005). Prevalence of the metabolic syndrome in patients with schizophrenia: baseline results from the Clinical Antipsychotic Trials of Intervention Effectiveness (CATIE) schizophrenia trial and comparison with national estimates from NHANES III. Schizophrenia Research 80, 19-32.

Meyer JM, Nasrallah HA, McEvoy JP, Goff DC, Davis SM, Chakos M, Patel JK, Keefe RS, Stroup TS, Lieberman JA (2005). The Clinical Antipsychotic Trials of Intervention Effectiveness (CATIE) Schizophrenia Trial: clinical comparison of subgroups with and without the metabolic syndrome. Schizophrenia Research 80, 9-18.

Mitchell AJ, Vancampfort D, Sweers K, van Winkel R, Yu W, De Hert M (2013). Prevalence of metabolic syndrome and metabolic abnormalities in schizophrenia and related 
disorders - a systematic review and meta-analysis. Schizophrenia Bulletin 39, 306-318.

Montgomery SA, Åsberg M (1979). A new depression scale designed to be sensitive to change. British Journal of Psychiatry 134, 382-389.

Morgan VA, McGrath JJ, Jablensky A, Badcock JC, Waterreus A, Bush R, Carr V, Castle D, Cohen M, Galletly C, Harvey C, Hocking B, McGorry P, Neil AL, Saw S, Shah S, Stain HJ, Mackinnon A (2014). Psychosis prevalence and physical, metabolic and cognitive co-morbidity: data from the Second Australian National Survey of Psychosis. Psychological Medicine 44, 2163-2176.

NAS (2012). Report of the National Audit of Schizophrenia. Healthcare Quality Improvement Partnership, Royal College of Psychiatrists: London.

Nasrallah HA, Meyer JM, Goff DC, McEvoy JP, Davis SM, Stroup TS, Lieberman JA (2006). Low rates of treatment for hypertension, dyslipidemia and diabetes in schizophrenia: data from the CATIE schizophrenia trial sample at baseline. Schizophrenia Research 86, 15-22.

National Institute of Health and Clinical Excellence (2014). National Institute of Health and Clinical Excellence: Core Interventions in the Treatment and Management of Schizophrenia in Primary and Secondary Care. NICE: London.

Nielsen J, Skadhede S, Correll CU (2010). Antipsychotics associated with the development of type 2 diabetes in antipsychotic-naive schizophrenia patients.

Neuropsychopharmacology 35, 1997-2004.

O'Brien C, Gardner-Sood P, Corlett SK, Ismail K, Smith S, Atakan Z, Greenwood K, Joseph C, Gaughran F (2014). Provision of health promotion programmes to people with serious mental illness: a mapping exercise of four South London boroughs. Journal of Psychiatric and Mental Health Nursing 21, 121-127.

Osborn DJ, Levy G, Nazareth I, Petersen I, Islam A, King MB (2007). Relative risk of cardiovascular and cancer mortality in people with severe mental illness from the United Kingdom's general practice research database. Archives of General Psychiatry 64, 242-249.

Paffenbarger RS Jr, Hyde RT, Wing AL, Lee IM, Jung DL, Kampert JB (1993). The association of changes in physical-activity level and other lifestyle characteristics with mortality among men. New England Journal of Medicine 328, 538-545.

Reilly S, Planner C, Hann M, Reeves D, Nazareth I, Lester H (2012). The role of primary care in service provision for people with severe mental illness in the United Kingdom. PLOS ONE 7, e36468.

Roe L, Strong C, Whiteside C, Neil A, Mant D (1994). Dietary intervention in primary care: validity of the DINE method for diet assessment. Family Practice 11, 375-381.

Rostami-Hodjegan A, Amin AM, Spencer EP, Lennard MS, Tucker GT, Flanagan RJ (2004). Influence of dose, cigarette smoking, age, sex, and metabolic activity on plasma clozapine concentrations: a predictive model and nomograms to aid clozapine dose adjustment and to assess compliance in individual patients. Journal of Clinical Psychopharmacology 24, 70-78.

Saha S, Chant D, McGrath J (2007). A systematic review of mortality in schizophrenia: is the differential mortality gap worsening over time? Archives of General Psychiatry 64, 1123-1131.

Saunders JB, Aasland OG, Babor TF, DeLaFuente JR, Grant M (1993). Development of the Alcohol Use Disorders Identification Test (AUDIT): WHO collaborative project on early detection of persons with harmful alcohol consumption - II. Addiction 88, 617-629.

Schizophrenia Commission (2012). The Abandoned Illness: A Report by the Schizophrenia Commission. Rethink Mental Illness: London.

Sobell L, Sobell M (1992). Timeline Follow-Back. In Measuring Alcohol Consumption (ed. R. Litten and J. Allen), pp. 41-72. Humana Press: New York.

The International Expert Committee (2009). International Expert Committee report on the role of the A1C assay in the diagnosis of diabetes. Diabetes Care 32, 1327-1334.

Tiihonen J, Lonnqvist J, Wahlbeck K, Klaukka T, Niskanen L, Tanskanen A, Haukka J (2009). 11-Year follow-up of mortality in patients with schizophrenia: a population-based cohort study (FIN11 study). Lancet 374, 620-627.

Vancampfort D, Knapen J, Probst M, Scheewe T, Remans S, De Hert M (2012). A systematic review of correlates of physical activity in patients with schizophrenia. Acta Psychiatrica Scandinavica 125, 352-362.

Vancampfort D, Knapen J, Probst M, van Winkel R, Deckx S, Maurissen K, Peuskens J, De Hert M (2010). Considering a frame of reference for physical activity research related to the cardiometabolic risk profile in schizophrenia. Psychiatry Research 177, 271-279.

Vancampfort D, Vansteelandt K, Correll CU, Mitchell AJ, De Herdt A, Sienaert P, Probst M, De Hert M (2013a). Metabolic syndrome and metabolic abnormalities in bipolar disorder: a meta-analysis of prevalence rates and moderators. American Journal of Psychiatry 170, 265-274.

Vancampfort D, Wampers M, Mitchell AJ, Correll CU, De Herdt A, Probst M, De Hert M (2013b). A meta-analysis of cardio-metabolic abnormalities in drug naive, first-episode and multi-episode patients with schizophrenia versus general population controls. World Psychiatry 12, 240-250.

van Winkel R, De Hert M, Van Eyck D, Hanssens L, Wampers M, Scheen A, Peuskens J (2008). Prevalence of diabetes and the metabolic syndrome in a sample of patients with bipolar disorder. Bipolar Disorders 10, 342-348.

Walker E, McGee RE, Druss BG (2015). Mortality in mental disorders and global disease burden implications: a systematic review and meta-analysis. JAMA Psychiatry. Published online 11 February 2015. doi:10.1001/ jamapsychiatry.2014.2502.

Zimmet P, Alberti KG, Shaw J (2001). Global and societal implications of the diabetes epidemic. Nature 414, 782-787. 